\title{
Competencias comunicativas de maestros en formación de educación especial
}

\section{José Luis Gallego-Ortega}

Universidad de Granada (España)

jlgalleg@ugr.es

\section{Antonio Rodríguez-Fuentes}

Universidad de Granada (España) arfuente@ugr.es

\section{Resumen}

El artículo presenta una investigación cuyo objetivo es conocer las percepciones de los estudiantes de magisterio sobre su competencia comunicativa. Fueron encuestados 115 estudiantes universitarios del grado de Maestro de Educación Primaria (concretamente de la mención especifica en Educación Especial) de la Universidad de Granada (España). Los resultados han revelado insuficiencias comunicativas relevantes para el desempeño de la actividad docente, así como un tímido incremento de ciertas habilidades comunicativas en el transcurso de la carrera.

\section{Palabras clave}

Competencias comunicativas, formación inicial, comunicación educativa, estudiantes de educación especial, Universidad de Granada-España. (Fuente: Tesauro de la Unesco).

Recepción: 2014-09-15 | Envío a pares: 2015-01-24 | Aceptación por pares: 2015-05-26 | Aprobación: 2015-06-02 DOI: 10.5294/edu.2015.18.2.2

Para citar este artículo / To reference this article / Para citar este artigo

Gallego-Ortega, J. L. y Rodríguez-Fuentes, A. (2015). Competencias comunicativas de maestros en formación de educación especial. Educ. Educ., 18 (2), 209-225. DOI: 10.5294/edu.2015.18.2.2 


\title{
The Communication Skills of Teachers in Special Education Training
}

\begin{abstract}
A study on student teachers' perceptions of their communication skills is described in this article. The sample included 115 students at the Universidad de Granada (Spain) who were candidates for a Master's Degree in Primary Education (majoring specifically in special education). The results showed significant shortcomings in communication that are relevant to teaching, as well as a slight increase in certain communication skills during the course of their studies.
\end{abstract}

Keywords

Communication skills, initial training, educational communication, special education students, Universidad de Granada-Spain (Source: Unesco Thesaurus). 


\section{Competências comunicativas de professores em formação de educação especial}

Resumo

Este artigo apresenta uma pesquisa cujo objetivo é conhecer as percepções dos estudantes de magistério sobre sua competência comunicativa. Foram entrevistados 115 estudantes universitários da graduação de "Magistério em Educação Primária" (especificamente em Educação Especial) da Universidad de Granada (Espanha). Os resultados revelaram insuficiências comunicativas relevantes para o desempenho da atividade docente, bem como um tênue aumento de certas habilidades comunicativas no transcurso da formação.

\section{Palavras chave}

Competências comunicativas, formação inicial, comunicação educativa, estudantes de educação especial, Universidad de Granada - Espanha. (Fonte: Tesauro da Unesco). 


\section{Introducción}

Con la Declaración de Bolonia, en 1999, y el Proyecto ALFA Tuning-América Latina, en 2003, Europa y América Latina inician un proceso de cambio en la enseñanza superior, uno de cuyos objetivos es diseñar un currículo por competencias. El término competencias, no obstante, posee un carácter anfibológico, dada su polisemia (Mérida, 2013), multidimensionalidad (Marciales, González, Castañeda y Barbosa, 2008) y perspectiva híbrida (Díaz, 2006). Sin embargo, de acuerdo con Perrenoud (2004), las competencias se entienden como la capacidad para movilizar recursos cognitivos y hacer frente a determinado tipo de situaciones de forma eficaz, es decir, las personas desarrollan competencias si resuelven problemas del entorno con idoneidad, mejora continua y ética (Tobón, 2012).

El enfoque basado en competencias demanda un profesional competente, capaz de gestionar su aula, un buen comunicador, un sujeto estratégico, habilidades sencillas de escribir pero difíciles de poseer (Gutiérrez, 2011). En el aprendizaje centrado en competencias, "se da una construcción en espiral en la acción, donde los conocimientos se perciben como herramientas útiles para la resolución de problemas" (Díaz, 2010, p. 46). Es comprensible, pues, que se demande un nuevo modelo de enseñanza universitaria centrada en el estudiante, "con contenidos, métodos, prácticas y medios de transmisión del saber que permitan superar la exclusividad del dominio cognitivo de las disciplinas, facilitandola adquisición de conocimientos prácticos, competencias y aptitudes para la comunicación" (Camargo y Pardo, 2008, p. 447).

Por su parte, la competencia comunicativa se entiende como la "capacidad de una persona para producir y comprender mensajes adecuados, tanto en el contexto de las temáticas tratadas como en las distintas situaciones comunicativas"(Viney Ferreira, 2012, p.139).Es, sin duda, una competencia básica para el profesorado (Perrenoud, 2004; Díaz, 2006; Mérida, 2013) y una de las competencias genéricas más citadas en la bibliografía especializada (Clemente y Escribá, 2013). Los maestros son comunicadores y sus logros dependen directamente de su habilidad para comunicar ideas, actitudes y valores (Ferreiro, 2011), aunque, como señalan Camacho y Sáenz (2000), no son pocos los docentes con problemas para comunicar o comunicarse.

En este contexto, nos planteamos la siguiente cuestión: ¿los futuros docentes de educación especial (en adelante EE) poseen las competencias comunicativas necesarias para responder a las necesidades inherentes a su profesión? Para ello se realizó un estudio con estudiantes del grado de Magisterio (mención en EE), con la intención de: 1) conocer su percepción acerca de su formación en competencias comunicativas; 2) analizar su posible progresión durante la carrera; 3) sugerir algunas estrategias para optimizar el desarrollo de esta competencia en la formación inicial.

\section{Método}

Se ha utilizado la denominada "investigación social mediante encuesta" (McMillan y Schumacher, 2005), para obtener datos de las declaraciones escritas de los estudiantes (futuros maestros de EE) sobre su competencia comunicativa.

La población objeto de estudio fue de 164 estudiantes (79,4\% mujeres y 20,6\% hombres) del grado de Maestro de Educación Primaria (mención en EE) de la Universidad de Granada (España). El número de sujetos encuestados se calculó mediante muestreo probabilístico. Para obtener el tamaño óptimo de la muestra en poblaciones finitas, que es el caso, se siguió el procedimiento referenciado por distintos autores (Latorre, Del Rincón y Arnal, 1996; Martínez, 2012), con un valor para $p=0,5$ y un nivel de confianza del 95\%. La muestra final estuvo configurada por 115 sujetos, que fueron encuestados en $2^{\circ}$ y $4^{\circ}$ curso.

Para obtener los datos, se utilizó un cuestionario (Apéndice I) que consta de 5 bloques temáticos 
(emisor, receptor, comunicador en el aula, participante en reuniones y tutor) y 60 ítems, utilizado en otras investigaciones que indagaron sobre la formación de diplomados universitarios en competencias comunicativas (Domingo, Gallego, García y Rodrí guez, 2010a; Domingo et al., 2010b; Domingo et al., 2010c). Se manejó una escala tipo Likert: dominio nulo (1), dominio insuficiente (2), dominio suficiente (3), dominio elevado (4), dominio muy elevado (5).

Este instrumento se sometió inicialmente al juicio de expertos y triangulación (Fox, 1981), para determinar la validez de su contenido. Luego, se validó de forma estadística, comprobando su fiabilidad y consistencia interna: $(\alpha=0,936$, coeficiente de fiabilidad de los ítem entre 0,935 y 0,936); prueba de las dos mitades de Guttman $\left(\alpha 1^{\mathrm{a}}\right.$ parte $=0,900, \alpha 2^{\mathrm{a}}$ parte $=0,881$; coeficiente de Spearman-Brown: 0,866), prueba KMO (índice significativo de 0,91 , próximo a la unidad) y prueba de Bartlett (chi-cuadrado de 19313,955) y muy alta significación ( $p=0,000$ ). El análisis de los datos cuantitativos se realizó con el Programa SPSS 18,0.

\section{Resultados}

Se presentan los resultados agrupados en los cinco bloques en los que se consolida la competencia comunicativa del profesorado (Camacho y Sáez, 2000).

\section{Resultados sobre la competencia como emisores}

En general, los alumnos admiten un bajo desarrollo de la habilidad como emisores (tabla 1), puesto que el valor preferentemente elegido (2) indica un dominio insuficiente de esta habilidad, como se aprecia en la moda, y con porcentajes relevantes. Se obtiene una media global cercana a ese mismo dominio (valor 2,4), salvo en los ítems 10 y 11 cuya moda se sitúa en el valor 3 (suficiente) claramente (43,2 y 40,5\%, respectivamente) y la media apunta hacia ese mismo valor $(2,9$ y 2,8 , respectivamente), lo que significa un desarrollo normal de esta habilidad. Mención especial requiere el ítem 9: si bien la moda es 2 (insuficiente), la media tiende hacia el valor $3(2,6)$, debido al elevado número de respuestas que se concentran en este valor (suficiente).

Tabla 1. Resultados globales sobre la habilidad como emisores

\begin{tabular}{|c|c|c|c|c|c|c|c|c|c|}
\hline \multirow{2}{*}{ Ítem } & \multicolumn{5}{|c|}{ Frecuencia de valores ( 1 a 5) } & \multicolumn{2}{|c|}{ Tendencia/Dispersión } & \multicolumn{2}{|c|}{ Preferencias } \\
\hline & 1 & 2 & 3 & 4 & 5 & Media & Desviación & Moda & $\%$ \\
\hline 1 & 23 & 99 & 23 & 3 & $\mathrm{O}$ & 2 & 0,6 & 2 & 66,9 \\
\hline 2 & 7 & 76 & 57 & 8 & $\mathrm{O}$ & 2,4 & 0,7 & 2 & 51,4 \\
\hline 3 & 18 & 69 & 48 & 8 & 5 & 2,4 & 0,9 & 2 & 46,6 \\
\hline 4 & 31 & 78 & 33 & 6 & $\mathrm{O}$ & 2,1 & 0,8 & 2 & 52,7 \\
\hline 5 & 45 & 53 & 38 & 10 & 2 & 2,1 & 1 & 2 & 35,8 \\
\hline 6 & 18 & 59 & 53 & 18 & $\mathrm{O}$ & 2,5 & 0,9 & 2 & 39,9 \\
\hline 7 & 8 & 74 & 50 & 15 & 1 & 2,5 & 0,8 & 2 & 50 \\
\hline 8 & 20 & 70 & 41 & 16 & 1 & 2,4 & 0,9 & 2 & 47,3 \\
\hline 9 & 19 & 53 & 52 & 22 & 2 & 2,6 & 0,9 & 2 & 35,8 \\
\hline 10 & 13 & 34 & 64 & 36 & 1 & 2,9 & 0,9 & 3 & 43,2 \\
\hline 11 & 6 & 56 & 60 & 21 & 5 & 2,8 & 0,9 & 3 & 40,5 \\
\hline 12 & 6 & 81 & 55 & 6 & O & 2,4 & 0,6 & 2 & 54,7 \\
\hline Total & & & & & & 2,4 & & 2 & \\
\hline
\end{tabular}


Atendiendo a los valores obtenidos por grupos (tabla 2), entre los alumnos de segundo curso predomina la moda insuficiente (2) junto a medias igualmente insuficientes (ítems 1, 4, 5, 8 y 12) o a medias entre suficientes e insuficientes $(2,6)$ (items 2, 7y 9), dado que en este último caso los porcentajes de aparición del valor modal (2) y su siguiente (3) resultan muy similares (44,9 y $44,5 \%$ para el ítem 2; 40,5 y 37,8\% para el ítem 7 y 36,2 y $34,7 \%$ para el ítem 9). Otro grupo (ítems 3, 6, 10 y 11) presenta también una media entre suficiente e insuficiente (entre 2,4 y 2,6) aunque su moda alcanza el valor 3 (suficiente). En suma, la habilidad como emisores en los alumnos de segundo se sitúa entre baja y normal, con tendencia a la baja, puesto que su media global es de 2,4 y predomina el valor modal 2.

Entre los alumnos de cuarto curso acontece algo distinto, dado que lo más frecuente en sus respuestas es una media suficiente (3) y una moda igual, como se aprecia en la mitad de los ítems (2, 3, $9,10,11$ y 12). Dos ítems más (6 y 7) presentan también una media que se acerca al valor 3 (suficiente) pero su moda es 2 (insuficiente), debido a que el porcentaje de aparición correspondiente a este valor modal (2) se acerca bastante al valor 3 (suficiente), como puede apreciarse en la tabla 2 (36,7 y $32,9 \%$ para el ítem 6 y 35,8 y 34\% para el ítem 7). También es insuficiente la moda de los ítems 4, 5 y 8, y además su media desciende oscilando entre los valores suficiente e insuficiente. En el ítem 1, sin embrago, la moda es suficiente y la media se sitúa entre los valores suficiente e insuficiente $(2,5)$. De lo anterior cabe inferir una variabilidad considerable entre las respuestas a los items sobre esta dimensión, que se agrupan en torno a una media general de 2,7 , lo que señala un desarrollo entre medio y bajo, aunque con predominio del valor modal 3 .

En definitiva, se aprecia una tímida evolución a favor del alumnado de $4^{\circ}$ en todas las dimensiones de la competencia como emisores, así como en sus valoraciones globales: la moda sube un punto (del valor 2 al 3) y la media 0,3 (de 2,4 a 2,7); la dispersión también aumenta en el último curso, aunque no sea significativa. En consecuencia, la valoración del progreso de esta competencia es negativa, a tenor de lo observado.

\section{Resultados sobre la competencia como receptores}

Los datos de la tabla 3 indican que, en general, la habilidad de los alumnos como receptores es suficiente, dada la prevalencia del valor que representa ese dominio $(\operatorname{moda}=3)$. La media global tiende también hacia ese mismo valor $(2,8)$, a excepción de los ítems 17, 18, 20 en los que aunque las medias se sitúan en torno al valor 2,5, la moda es 2 (insuficiente), y en el ítem 21, donde la moda es 2 pero el valor 3 , obtiene resultados muy similares (37,2 y $35,1 \%$, respectivamente). En estos últimos casos puede indicarse un desarrollo medio-bajo de la habilidad.

Por cursos, para los alumnos de $2^{\circ}$, los valores sobre su competencia como receptores contenidos en la tabla 4 (items 13, 14, 15, 16, 19 y 22) se agrupan en torno a una media suficiente (3), con una moda también suficiente, si bien en uno de ellos (item 13) el porcentaje de presencia del valor más elegido ( $\operatorname{moda}=3$ ) coincide con el valor anterior (2) (33,9 y 33,5\%, respectivamente). Se observa un grupo menor de ítems (17, 20 y 21) cuya moda es 2 (insuficiente) y su media ronda el valor 2,5 , es decir, entre suficiente e insuficiente. Con fundamento en lo anterior y los valores relativamente bajos de la dispersión de datos, puede generalizarse una media con un valor global de 2,7 y una moda predominantemente suficiente (3).

De otra parte, los alumnos del último curso muestran más claramente un desarrollo aceptable de esta competencia, corroborado con la presencia mayoritaria de una moda y una media en torno al valor 3 (dominio suficiente), salvo en dos de los items (13 y 14) que se caracterizan estadísticamente por una moda elevada (4) y una media entre sufi- 
Tabla 2. Resultados por cursos sobre la habilidad como emisores

\begin{tabular}{|c|c|c|c|c|c|c|c|c|c|}
\hline \multirow{2}{*}{ Ítem } & \multirow{2}{*}{ Curso } & \multicolumn{5}{|c|}{ Porcentaje de valores ( 1 a 5 ) } & \multicolumn{3}{|c|}{ Tendencia/Dispersión } \\
\hline & & 1 & 2 & 3 & 4 & 5 & Moda & Media & Desviación \\
\hline \multirow{2}{*}{1} & $2^{\circ}$ & 17,1 & 55,1 & 26 & 1,7 & $\mathrm{O}$ & 2 & 2,1 & 0,7 \\
\hline & $4^{\circ}$ & 11,6 & 35 & 40,4 & 12,9 & $\mathrm{O}$ & 3 & 2,5 & 0,9 \\
\hline \multirow{2}{*}{2} & $2^{\circ}$ & 5,6 & 44,9 & 44,5 & 3,1 & 1,9 & 2 & 2,6 & 0,7 \\
\hline & $4^{\circ}$ & 3,2 & 30,2 & 39,9 & 25,1 & 1,6 & 3 & 2,9 & 0,9 \\
\hline \multirow{2}{*}{3} & $2^{\circ}$ & 17,7 & 33,1 & 41,8 & 5,8 & 1,5 & 3 & 2,4 & 1 \\
\hline & $4^{0}$ & 4 & 31,3 & 38 & 24 & 2,7 & 3 & 2,9 & 0,9 \\
\hline \multirow{2}{*}{4} & $2^{0}$ & 20,4 & 50,9 & 24,5 & 4,3 & 0 & 2 & 2,1 & 0,9 \\
\hline & $4^{0}$ & 11,6 & 43,1 & 37,5 & 7,5 & 0,3 & 2 & 2,4 & 0,8 \\
\hline \multirow{2}{*}{5} & $2^{0}$ & 29,1 & 33,3 & 27 & 10,2 & 0,4 & 2 & 2,2 & 1 \\
\hline & $4^{\circ}$ & 19,9 & 41 & 27 & 11,1 & 1,1 & 2 & 2,3 & 1 \\
\hline \multirow{2}{*}{6} & $2^{\circ}$ & 11,8 & 35,1 & 40,8 & 11,6 & 0,8 & 3 & 2,6 & 0,9 \\
\hline & $4^{\circ}$ & 8,9 & 36,7 & 32,9 & 20,8 & 0,8 & 2 & 2,7 & 0,9 \\
\hline \multirow{2}{*}{7} & $2^{0}$ & 9,2 & 40,5 & 37,8 & 12,1 & 0,4 & 2 & 2,6 & 0,8 \\
\hline & $4^{\circ}$ & 10 & 35,8 & 34 & 17,5 & 2,7 & 2 & 2,7 & 1 \\
\hline \multirow{2}{*}{8} & $2^{\circ}$ & 17,9 & 46,6 & 29,5 & 6 & 0 & 2 & 2,2 & 0,8 \\
\hline & $4^{\circ}$ & 11,1 & 40,4 & 31,8 & 15,4 & 1,3 & 2 & 2,6 & 0,9 \\
\hline \multirow{2}{*}{9} & $2^{\circ}$ & 11,9 & 36,2 & 34,7 & 16,4 & 0,8 & 2 & 2,6 & 0,9 \\
\hline & $4^{\circ}$ & 8,9 & 32,6 & 35,8 & 19,4 & 3,2 & 3 & 2,8 & 1 \\
\hline \multirow{2}{*}{10} & $2^{\circ}$ & 5,6 & 2,2 & 52,2 & 21,2 & 0,8 & 3 & 2,6 & 0,8 \\
\hline & $4^{\circ}$ & 2,2 & 21,8 & 43,7 & 29,4 & 3 & 3 & 3,1 & 0,9 \\
\hline \multirow{2}{*}{11} & $2^{0}$ & 3,9 & 32,9 & 43,9 & 17,7 & 1,5 & 3 & 2,4 & 0,9 \\
\hline & $4^{\circ}$ & 3,8 & 19,1 & 45,3 & 29,1 & 2,7 & 3 & 3,1 & 0,9 \\
\hline \multirow{2}{*}{12} & $2^{\circ}$ & 6 & 49,7 & 40,3 & 3,3 & 0,8 & 2 & 2,4 & 0,7 \\
\hline & $4^{\circ}$ & 5,4 & 35,8 & 40,4 & 17,8 & 0,5 & 3 & 2,7 & 0,8 \\
\hline
\end{tabular}

ciente y elevada (en torno al valor 3,5), aunque en el ítem 14 la moda presenta un porcentaje de aparición similar al del valor anterior ( 41,8 y $38,3 \%$, respectivamente). En consecuencia, la media total para esta competencia es de 3,1 y la moda es el valor 3, siendo la desviación poco significativa, con valores en torno al 1.

Se aprecia, pues, un ligero avance en la competencia como receptores, puesto que si bien la moda de ambos grupos coincide en el valor 3 (suficiente), la media evidencia el sutil avance logrado: 2,7 en $2^{\circ}$ curso y $3,1 \mathrm{en} 4^{\circ}$. El avance se aprecia para todos los ítems, sin que la dispersión de un grupo sea mayor que la del otro, siendo en todos los casos ínfima, por debajo de 1. Cabe concluir que, aunque se aprecia cierta progresión, se nos antoja escasa a la luz de su trascendencia.

\section{Resultados sobre la competencia para comunicar en el aula}

La media global $(2,4)$ y la representación de la moda (2) denotan un dominio insuficiente de esta habilidad. En efecto, una docena de ítems conforma el grupo mayoritario que vertebra esta habilidad valorada deficientemente (media y modas insuficientes, 
ISSN $0123-1294$ | Educ.Educ. Vol. 18. No. 2 | Mayo-Agosto de 2015 | pp. 209-225.

Universidad de La Sabana | Facultad de Educación

Tabla 3. Resultados globales sobre la habilidad como receptores

\begin{tabular}{|c|c|c|c|c|c|c|c|c|c|}
\hline \multirow{2}{*}{ Ítem } & \multicolumn{4}{|c|}{ Frecuencia de valores } & \multicolumn{2}{c|}{ Tendencia/Dispersión } & \multicolumn{2}{c|}{ Preferencias } \\
\cline { 2 - 11 } & 1 & 2 & 3 & 4 & 5 & Media & Desviación & Moda & $\%$ \\
\hline 13 & 5 & 30 & 59 & 51 & 3 & 3,1 & 0,9 & 3 & 39,9 \\
\hline 14 & 0 & 50 & 59 & 38 & 1 & 2,9 & 1 & 3 & 39,9 \\
\hline 15 & 9 & 42 & 68 & 27 & 2 & 2,8 & 0,9 & 3 & 45,9 \\
\hline 16 & 13 & 47 & 60 & 28 & 0 & 2,8 & 0,9 & 3 & 40,5 \\
\hline 17 & 14 & 65 & 55 & 12 & 2 & 2,5 & 0,8 & 2 & 43,9 \\
\hline 18 & 10 & 66 & 55 & 16 & 1 & 2,5 & 0,8 & 2 & 44,6 \\
\hline 19 & 7 & 44 & 63 & 32 & 2 & 2,9 & 0,9 & 3 & 42,6 \\
\hline 20 & 21 & 62 & 40 & 20 & 5 & 2,6 & 1 & 2 & 41,9 \\
\hline 21 & 16 & 55 & 52 & 24 & 1 & 2,6 & 0,9 & 2 & 37,2 \\
\hline 22 & 5 & 56 & 60 & 23 & 4 & 2,8 & 0,9 & 3 & 40,5 \\
\hline Total & & & & & & 2,8 & & 3 & 41,7 \\
\hline
\end{tabular}

Tabla 4. Resultados por cursos sobre la habilidad como receptores

\begin{tabular}{|c|c|c|c|c|c|c|c|c|c|}
\hline \multirow{2}{*}{ Ítem } & \multirow{2}{*}{ Curso } & \multicolumn{5}{|c|}{ Porcentaje de valores ( 1 a 5 ) } & \multicolumn{3}{|c|}{ Tendencia/Dispersión } \\
\hline & & 1 & 2 & 3 & 4 & 5 & Moda & Media & Desviación \\
\hline \multirow{2}{*}{13} & $2^{\circ}$ & 1,9 & 33,5 & 33,9 & 27,7 & 2,9 & 3 & 3 & 0,9 \\
\hline & $4^{\circ}$ & 1,9 & 14 & 31,3 & 47,4 & 5,4 & 4 & 3,4 & 0,9 \\
\hline \multirow{2}{*}{14} & $2^{\circ}$ & 5,4 & 32,2 & 43 & 19,5 & O & 3 & 2,8 & 0,8 \\
\hline & $4^{\circ}$ & 0,5 & 14,8 & 38,3 & 41,8 & 4,6 & 4 & 3,4 & 0,8 \\
\hline \multirow{2}{*}{15} & $2^{\circ}$ & 7,7 & 28,7 & 42,2 & 20,4 & 1 & 3 & 2,8 & 0,9 \\
\hline & $4^{\circ}$ & 2,4 & 12,9 & 44,2 & 35,3 & 5,1 & 3 & 3,3 & 0,8 \\
\hline \multirow{2}{*}{16} & $2^{\circ}$ & 10 & 28,9 & 44,1 & 17 & 0 & 3 & 2,7 & 0,9 \\
\hline & $4^{\circ}$ & 4,6 & 18,9 & 43,7 & 31 & 1,9 & 3 & 3,1 & 0,9 \\
\hline \multirow{2}{*}{17} & $2^{0}$ & 10,2 & 55,5 & 24,3 & 9,6 & 0,4 & 2 & 2,4 & 0,8 \\
\hline & $4^{\circ}$ & 5,4 & 28,3 & 42,9 & 22,6 & 0,8 & 3 & 2,9 & 0,9 \\
\hline \multirow{2}{*}{18} & $2^{\circ}$ & 7,3 & 33,9 & 49,1 & 8,9 & 0,8 & 3 & 2,6 & 0,8 \\
\hline & $4^{\circ}$ & 2,7 & 32,6 & 39,1 & 23,2 & 2,4 & 3 & 2,9 & 0,9 \\
\hline \multirow{2}{*}{19} & $2^{\circ}$ & 3,3 & 35,5 & 45,1 & 14,6 & 1,5 & 3 & 2,8 & 0,8 \\
\hline & $4^{\circ}$ & 2,4 & 19,9 & 43,4 & 31,3 & 3 & 3 & 3,1 & 0,9 \\
\hline \multirow{2}{*}{20} & $2^{0}$ & 15 & 40,1 & 35,5 & 9,1 & 0,4 & 2 & 2,4 & 0,9 \\
\hline & $4^{\circ}$ & 10,5 & 25,9 & 36,1 & 24,3 & 3,2 & 3 & 2,8 & 1 \\
\hline \multirow{2}{*}{21} & $2^{0}$ & 10,8 & 43,7 & 33,1 & 11,9 & 0,4 & 2 & 2,5 & 0,9 \\
\hline & $4^{\circ}$ & 7 & 24,5 & 39,1 & 26,1 & 3,2 & 3 & 2,9 & 1 \\
\hline \multirow{2}{*}{22} & $2^{0}$ & 2,5 & 34,5 & 48,7 & 13,5 & 0,8 & 3 & 2,8 & 0,8 \\
\hline & $4^{\circ}$ & 3,2 & 15,4 & 41,2 & 37,2 & 3 & 3 & 3,2 & 0,9 \\
\hline
\end{tabular}


valor 2). Tres ítems más (29, 33 y 39) reflejan una media entre insuficiente y suficiente (en torno al 2,5) y una moda insuficiente (2); otros tantos $(26,28,32$ y 34) obtienen una valoración entre insuficiente y suficiente, pero esta vez con una moda suficiente (3); mientras que el ítem 41 presenta una media suficiente $(2,9)$ y una moda casi idéntica (3). De manera que la media global se sitúa entre los valores insuficiente y suficiente $(2,4)$ y la moda más frecuente se corresponde con el valor 2 (insuficiente), con unos niveles de dispersión poco acusados (tabla 5).

Teniendo en cuenta el curso (tabla 6), el alumnado de $2^{\circ}$ alcanza apenas una moda insuficiente (2), con una media que oscila entre insuficiente y suficiente (en torno al 2,5) (ítems 25, 26, 27, 28, 31, 33, 35,
$36,39,40$ y 41), si bien en 4 de ellos el porcentaje de la moda es parecido al del valor superior (40,5 y $38,9 \%$ para el ítem 33; 42,6 y $38,7 \%$ para el ítem $39 ; 43,9 \%$ y 41 para el ítem 40 y 34,1\% y 33,3\% para el ítem 41 ). A este grupo le sigue otro numeroso (ítems 23, 24, 30, 37, 38 y 42) que presenta también una moda insuficiente (2) y una media igualmente insuficiente. Por otro lado, se encuentran algunos ítems cuya moda es suficiente (3) y su media también (ítem 34), o entre suficiente e insuficiente (ítems 29 y 32). El valor promedio de las medias parciales es 2,3 (insuficiente) y su valor modal predominante también lo es (2), de lo que se infiere un bajo dominio de esta habilidad en los alumnos encuestados. La dispersión no es apreciable, lo que corrobora la conclusión anterior.

Tabla 5. Resultados globales sobre la habilidad como comunicadores en el aula

\begin{tabular}{|c|c|c|c|c|c|c|c|c|c|}
\hline \multirow{2}{*}{ Ítem } & \multicolumn{5}{|c|}{ Frecuencia de valores } & \multicolumn{2}{|c|}{ Tendencia/Dispersión } & \multicolumn{2}{|c|}{ Preferencias } \\
\hline & 1 & 2 & 3 & 4 & 5 & Media & Desviación & Moda & $\%$ \\
\hline 23 & 20 & 75 & 43 & 9 & 1 & 2,3 & 0,8 & 2 & 50,6 \\
\hline 24 & 8 & 81 & 45 & 14 & $\mathrm{O}$ & 2,4 & 0,7 & 2 & 54,7 \\
\hline 25 & 14 & 72 & 55 & 7 & $\mathrm{O}$ & 2,4 & 0,7 & 2 & 48,6 \\
\hline 26 & 27 & 43 & 58 & 19 & 1 & 2,5 & 1 & 3 & 39,1 \\
\hline 27 & 15 & 65 & 59 & 9 & O & 2,4 & 0,8 & 2 & 43,9 \\
\hline 28 & 21 & 51 & 58 & 17 & 1 & 2,5 & 0,9 & 3 & 39,1 \\
\hline 29 & 10 & 62 & 52 & 24 & O & 2,6 & 0,8 & 2 & 41,8 \\
\hline 30 & 21 & 72 & 44 & 10 & 1 & 2,3 & 0,8 & 2 & 48,6 \\
\hline 31 & 14 & 65 & 54 & 13 & 2 & 2,5 & 0,8 & 2 & 43,9 \\
\hline 32 & 20 & 48 & 62 & 17 & 1 & 2,5 & 0,9 & 3 & 41,8 \\
\hline 33 & 14 & 71 & 45 & 17 & 1 & 2,5 & 0,8 & 2 & 48 \\
\hline 34 & 13 & 54 & 60 & 16 & 5 & 2,6 & 0,9 & 3 & 40,5 \\
\hline 35 & 16 & 69 & 46 & 17 & 0 & 2,4 & 0,8 & 2 & 46,6 \\
\hline 36 & 17 & 67 & 50 & 12 & 1 & 2,4 & 0,8 & 2 & 45,3 \\
\hline 37 & 29 & 64 & 45 & 10 & 0 & 2,2 & 0,8 & 2 & 43,2 \\
\hline 38 & 32 & 60 & 44 & 12 & 0 & 2,2 & 0,7 & 2 & 40,6 \\
\hline 39 & 15 & 64 & 52 & 16 & 1 & 2,5 & 0,9 & 2 & 43,2 \\
\hline 40 & 10 & 75 & 51 & 11 & 1 & 2,4 & 0,8 & 2 & 50,7 \\
\hline 41 & 4 & 43 & 63 & 35 & 3 & 2,9 & 1 & 3 & 42,6 \\
\hline 42 & 15 & 71 & 47 & 13 & 2 & 2,4 & 0,8 & 2 & 48 \\
\hline Total & & & & & & 2,4 & & 2 & \\
\hline
\end{tabular}


ISSN 0123-1294 | Educ.Educ. Vol. 18. No. 2 | Mayo-Agosto de 2015 | pp. 209-225.

Universidad de La Sabana | Facultad de Educación

Tabla 6. Resultados por cursos sobre la habilidad como comunicadores en el aula

\begin{tabular}{|c|c|c|c|c|c|c|c|c|c|}
\hline \multirow{2}{*}{ Ítem } & \multirow{2}{*}{ Curso } & \multicolumn{5}{|c|}{ Porcentaje de valores ( 1 a 5 ) } & \multicolumn{3}{|c|}{ Tendencia/Dispersión } \\
\hline & & 1 & 2 & 3 & 4 & 5 & Moda & Media & Desviación \\
\hline \multirow{2}{*}{23} & $2^{\circ}$ & 12,1 & 54,5 & 30,6 & 2,7 & 0 & 2 & 2,2 & 0,7 \\
\hline & $4^{\circ}$ & 5,1 & 28,3 & 48 & 18,1 & 0,5 & 3 & 2,8 & 0,8 \\
\hline \multirow{2}{*}{24} & $2^{\circ}$ & 14,6 & 53,6 & 29,1 & 2,7 & o & 2 & 2,2 & 0,7 \\
\hline & $4^{\circ}$ & 5,9 & 35 & 45,8 & 13,2 & 0 & 3 & 2,7 & 0,8 \\
\hline \multirow{2}{*}{25} & $2^{\circ}$ & 6,9 & 54,3 & 31,6 & 6,4 & 0,8 & 2 & 2,4 & 0,7 \\
\hline & $4^{\circ}$ & 6,5 & 31 & 43,7 & 18,6 & 0,3 & 3 & 2,8 & 0,8 \\
\hline \multirow{2}{*}{26} & $2^{\circ}$ & 10,6 & 39,3 & 36,8 & 13,3 & 0 & 2 & 2,5 & 0,9 \\
\hline & $4^{\circ}$ & 6,7 & 30,2 & 37,5 & 24,8 & 0,8 & 3 & 2,8 & 0,9 \\
\hline \multirow{2}{*}{27} & $2^{\circ}$ & 10,8 & 42,8 & 32,8 & 13,7 & 0 & 2 & 2,5 & 0,9 \\
\hline & $4^{\circ}$ & 5,9 & 32,1 & 37,7 & 23,7 & 0,5 & 3 & 2,8 & 0,9 \\
\hline \multirow{2}{*}{28} & $2^{\circ}$ & 13,5 & 41,6 & 37,2 & 7,7 & 0 & 2 & 2,4 & 0,8 \\
\hline & $4^{\circ}$ & 10,2 & 31 & 39,4 & 18,1 & 1,3 & 3 & 2,6 & 0,9 \\
\hline \multirow{2}{*}{29} & $2^{\circ}$ & 11 & 37,2 & 40,5 & 10,6 & 0,8 & 3 & 2,5 & 0,9 \\
\hline & $4^{\circ}$ & 6,5 & 27 & 36,4 & 28,3 & 1,9 & 3 & 2,9 & 0,9 \\
\hline \multirow{2}{*}{30} & $2^{\circ}$ & 18,7 & 48,7 & 27,7 & 4,8 & 0 & 2 & 2,2 & 0,8 \\
\hline & $4^{\circ}$ & 8,9 & 34,2 & 40,4 & 15,4 & 1,1 & 3 & 2,6 & 0,9 \\
\hline \multirow{2}{*}{31} & $2^{\circ}$ & 12,1 & 42,6 & 36,8 & 8,1 & 0,4 & 2 & 2,4 & 0,8 \\
\hline & $4^{\circ}$ & 7,3 & 28,3 & 38,3 & 23,5 & 2,2 & 3 & 2,8 & 0,9 \\
\hline \multirow{2}{*}{32} & $2^{\circ}$ & 11,8 & 37,8 & 41,6 & 7,5 & 1,3 & 3 & 2,5 & 0,8 \\
\hline & $4^{\circ}$ & 8,1 & 28 & 34,5 & 27,2 & 2,2 & 3 & 2,9 & 1 \\
\hline \multirow{2}{*}{33} & $2^{\circ}$ & 10 & 40,5 & 38,9 & 10,6 & 0 & 2 & 2,5 & 0,8 \\
\hline & $4^{\circ}$ & 7,8 & 26,4 & 44,7 & 19,4 & 1,6 & 3 & 2,8 & 0,9 \\
\hline \multirow{2}{*}{34} & $2^{\circ}$ & 7,7 & 30,1 & 40,1 & 21,4 & 0,8 & 3 & 2,8 & 0,9 \\
\hline & $4^{\circ}$ & 8,4 & 19,9 & 41 & 28,6 & 2,2 & 3 & 3 & 1 \\
\hline \multirow{2}{*}{35} & $2^{\circ}$ & 11,4 & 40,8 & 35,8 & 11,2 & 0,8 & 2 & 2,5 & 0,9 \\
\hline & $4^{\circ}$ & 10,5 & 34,5 & 30,5 & 24 & 0,5 & 2 & 2,7 & 1 \\
\hline \multirow{2}{*}{36} & $2^{\circ}$ & 8,7 & 47,8 & 37,6 & 6 & 0 & 2 & 2,4 & 0,7 \\
\hline & $4^{\circ}$ & 10,5 & 36,1 & 32,9 & 19,1 & 0,3 & 2 & 2,6 & 0,9 \\
\hline \multirow{2}{*}{37} & $2^{0}$ & 15,4 & 49,5 & 22,7 & 11,6 & 0,8 & 2 & 2,3 & 0,9 \\
\hline & $4^{\circ}$ & 10,2 & 29,6 & 40,2 & 17,8 & 2,2 & 3 & 2,7 & 0,9 \\
\hline \multirow{2}{*}{38} & $2^{\circ}$ & 21 & 36,8 & 32,9 & 9,2 & 0 & 2 & 2,3 & 0,9 \\
\hline & $4^{\circ}$ & 11,1 & 30,5 & 35,8 & 22,4 & 0,3 & 3 & 2,7 & 1 \\
\hline \multirow{2}{*}{39} & $2^{\circ}$ & 10,8 & 42,6 & 38,7 & 7,9 & 0 & 2 & 2,4 & 0,8 \\
\hline & $4^{\circ}$ & 6,2 & 31,5 & 32,6 & 18 & 1,6 & 3 & 2,5 & 0,9 \\
\hline \multirow{2}{*}{40} & $2^{\circ}$ & 7,1 & 43,9 & 41 & 7,9 & 0 & 2 & 2,5 & 0,7 \\
\hline & $4^{\circ}$ & 3,2 & 28 & 39,9 & 27 & 1,9 & 3 & 3 & 1 \\
\hline \multirow{2}{*}{41} & $2^{\circ}$ & 9,2 & 34,1 & 33,3 & 22,9 & 0,4 & 2 & 2,7 & 0,9 \\
\hline & $4^{\circ}$ & 3,8 & 23,7 & 36,7 & 33,2 & 2,7 & 3 & 3,1 & 0,9 \\
\hline & $2^{\circ}$ & 14,6 & 50,3 & 26,2 & 8,9 & 0 & 2 & 2,3 & 0,8 \\
\hline 42 & $4^{\circ}$ & 8,4 & 29,4 & 41,8 & 18,9 & 1,6 & 3 & 2,8 & 0,9 \\
\hline
\end{tabular}


En los alumnos de $4^{\circ}$ se aprecian diferencias. $\mathrm{Si}$ lo habitual es la presencia de una moda suficiente (3) junto a una media suficiente (ítems 23, 24, 25, 26, 27, 29, 31, 32, 33, 34, 38, 39, 40, 41 y 42), también se combina la moda suficiente con una media entre suficiente e insuficiente, en torno al valor 2,5 (ítems 28, 30 y 39), resaltando, asimismo, los valores del ítem 35 con una moda insuficiente (2) y una media entre suficiente e insuficiente. En definitiva, la media total $(2,8)$ y su moda (3) indican un dominio suficiente de esta competencia.

Es de resaltar el incremento que experimenta esta competencia de $2^{\circ}$ a $4^{\circ}$ : un punto en el valor modal, que pasa del dominio insuficiente al suficiente, y 0,5 en la media general, que pasa de 2,3 a 2,8, lo que significa sin duda un avance en su desarrollo, aunque limitado. Tampoco la dispersión merece ser resaltada puesto que los datos se presentan bastante agrupados en torno al valor 3 (suficiente).

\section{Resultados sobre la competencia para mantener reuniones con padres y compañeros}

En general, la agrupación de datos sitúa a esta habilidad en un nivel de dominio insuficiente, dado que la moda obtiene ese valor (2) y la media en torno al 2,5; salvo dos items: el 43, cuya moda es 3 y su media apunta hacia un valor suficiente $(2,9)$; y el 47 , que obtiene una media también suficiente $(2,8)$, pero la moda es 2, como en el caso de la mayoría de ítems de esta dimensión, aunque el valor 3 obtiene un porcentajecasiidéntico(31,8y31,1\%,respectivamente). En el item 45 acontece algo similar en cuanto a la moda, situada en el valor 2 pero empatada con el valor 3 (35,8 y 35,1\%, respectivamente), si bien en este caso la media es más baja $(2,4)$. En general, la media es 2,5 , entre suficiente e insuficiente, y la moda es insuficiente (2). El agrupamiento de datos alrededor de la media, calculado a través de la desviación típica, es relativamente notable, dado que la desviación es baja (tabla 7).

Tabla 7. Resultados globales sobre la habilidad como participantes en reuniones

\begin{tabular}{|c|c|c|c|c|c|c|c|c|c|}
\hline \multirow{2}{*}{ Ítem } & \multicolumn{5}{|c|}{ Frecuencia de valores } & \multicolumn{2}{|c|}{ Tendencia/Dispersión } & \multicolumn{2}{|c|}{ Preferencias } \\
\hline & 1 & 2 & 3 & 4 & 5 & Media & Desviación & Moda & $\%$ \\
\hline 43 & 4 & 45 & 70 & 27 & 2 & 2,9 & 0,8 & 3 & 47,3 \\
\hline 44 & 18 & 75 & 38 & 16 & 1 & 2,4 & 0,9 & 2 & 50,7 \\
\hline 45 & 25 & 53 & 52 & 16 & 2 & 2,4 & 0,9 & 2 & 35,8 \\
\hline 46 & 21 & 62 & 47 & 18 & $\mathrm{O}$ & 2,4 & 0,9 & 2 & 41,9 \\
\hline 47 & 12 & 47 & 46 & 41 & 2 & 2,8 & 1 & 2 & 31,8 \\
\hline 48 & 14 & 71 & 43 & 20 & $\mathrm{O}$ & 2,5 & 0,8 & 2 & 48 \\
\hline 49 & 20 & 65 & 48 & 14 & $\mathrm{O}$ & 2,4 & 0,8 & 2 & 43,9 \\
\hline 50 & 13 & 61 & 57 & 16 & 1 & 2,5 & 0,8 & 2 & 41,2 \\
\hline 51 & 10 & 69 & 50 & 17 & 2 & 2,5 & 0,8 & 2 & 46,6 \\
\hline 52 & 21 & 60 & 48 & 19 & O & 2,4 & 0,9 & 2 & 40,5 \\
\hline 53 & 14 & 65 & 52 & 15 & 2 & 2,5 & 0,9 & 2 & 43,9 \\
\hline 54 & 13 & 71 & 43 & 18 & 3 & 2,5 & 0,9 & 2 & 48 \\
\hline Total & & & & & & 2,5 & & 2 & \\
\hline
\end{tabular}


ISSN 0123-1294 | Educ.Educ. Vol. 18. No. 2 | Mayo-Agosto de 2015 | pp. 209-225.

Universidad de La Sabana | Facultad de Educación

Por cursos (tabla 8), los datos de $2^{\circ}$ reflejan que la mayoría de los ítems han sido autovalorados con una moda insuficiente y una media entre suficiente e insuficiente, principalmente (ítems 44, 46, 48, 49, 51, 52,53 y 54). El ítem 50 presenta una moda y una media insuficientes; el item 45 ha obtenido una media entre suficiente e insuficiente pero su moda es suficiente, aunque bien podría ser insuficiente puesto que el porcentaje correspondiente con este valor es casi idéntico al del valor de la moda $(36,2$ y $37,6 \%$, respectivamente). Por último, tan solo dos ítems ( 43 y 47) han alcanzado una moda y media suficientes. En consecuencia, cabe colegir un nivel medio-bajo de esta habilidad comunicativa, con una media absoluta de 2,5 y un valor preferente de 2 .

El alumnado de $4^{\circ}$, sin embargo, se autovalora en todos los ítems con un dominio suficiente, lo que se demuestra con el cálculo de su media global que alcanza el valor de 2,9, muy próximo al de la moda (3). La dispersión de datos no es considerable, dado que todos los ítems presentan valores muy similares.

Tabla 8. Resultados por cursos sobre la habilidad como participantes en reuniones

\begin{tabular}{|c|c|c|c|c|c|c|c|c|c|}
\hline \multirow{2}{*}{ Ítem } & \multirow{2}{*}{ Curso } & \multicolumn{5}{|c|}{ Porcentaje de valores ( 1 a 5 ) } & \multicolumn{3}{|c|}{ Tendencia/Dispersión } \\
\hline & & 1 & 2 & 3 & 4 & 5 & Moda & Media & Desviación \\
\hline \multirow{2}{*}{43} & $2^{\circ}$ & 6,9 & 30,1 & 41,2 & 19,8 & 1,9 & 3 & 2,8 & 0,9 \\
\hline & $4^{\circ}$ & 4,6 & 22,9 & 38,3 & 32,1 & 2,2 & 3 & 3 & 0,9 \\
\hline \multirow{2}{*}{44} & $2^{\circ}$ & 3,7 & 58,2 & 31,6 & 6,6 & $\mathrm{O}$ & 2 & 2,4 & 0,7 \\
\hline & $4^{\circ}$ & 6,2 & 30,5 & 36,1 & 25,6 & 1,6 & 3 & 2,9 & 0,9 \\
\hline \multirow[b]{2}{*}{45} & $2^{\circ}$ & 13,5 & 36,2 & 37,6 & 12,7 & 0 & 3 & 2,5 & 0,9 \\
\hline & $4^{\circ}$ & 6,2 & 26,1 & 42,6 & 22,9 & 2,2 & 3 & 2,9 & 0,9 \\
\hline \multirow{2}{*}{46} & $2^{\circ}$ & 9,4 & 46,4 & 34,7 & 7,9 & 0,8 & 2 & 2,4 & 0,8 \\
\hline & $4^{\circ}$ & 3,2 & 25,6 & 45,3 & 24,3 & 1,6 & 3 & 3 & 0,8 \\
\hline \multirow{2}{*}{47} & $2^{\circ}$ & 7,9 & 31 & 38 & 22,4 & 0,8 & 3 & 2,8 & 0,9 \\
\hline & $4^{\circ}$ & 3 & 24,3 & 36,1 & 31,3 & 5,1 & 3 & 3,1 & 0,9 \\
\hline \multirow{2}{*}{48} & $2^{\circ}$ & 11,6 & 46,4 & 32 & 10 & 0 & 2 & 2,4 & 0,8 \\
\hline & $4^{\circ}$ & 8,4 & 29,1 & 35 & 26,4 & 1,1 & 3 & 2,8 & 1 \\
\hline \multirow{2}{*}{49} & $2^{\circ}$ & 12,3 & 40,8 & 37,2 & 9,6 & 0 & 2 & 2,4 & 0,8 \\
\hline & $4^{\circ}$ & 7 & 28,3 & 41,8 & 22,4 & 0,5 & 3 & 2,8 & 0,9 \\
\hline \multirow{2}{*}{50} & $2^{\circ}$ & 11,4 & 50,5 & 32 & 6,2 & 0 & 2 & 2,3 & 0,8 \\
\hline & $4^{\circ}$ & 4,9 & 30,5 & 39,1 & 23,7 & 1,9 & 3 & 2,9 & 0,9 \\
\hline \multirow{2}{*}{51} & $2^{\circ}$ & 7,7 & 48,2 & 35,3 & 8,9 & $\mathrm{O}$ & 2 & 2,5 & 0,8 \\
\hline & $4^{\circ}$ & 4 & 27,2 & 43,9 & 23,5 & 1,3 & 3 & 2,9 & 0,9 \\
\hline \multirow{2}{*}{52} & $2^{\circ}$ & 11,8 & 43 & 38,2 & 7,1 & 0 & 2 & 2,4 & 0,8 \\
\hline & $4^{\circ}$ & 3,8 & 28,6 & 44,2 & 22,4 & 1,1 & 3 & 2,9 & 0,8 \\
\hline \multirow{2}{*}{53} & $2^{\circ}$ & 5,2 & 44,1 & 38,5 & 11,8 & 0,4 & 2 & 2,6 & 0,8 \\
\hline & $4^{\circ}$ & 4 & 26,7 & 40,7 & 26,1 & 2,4 & 3 & 3 & 0,9 \\
\hline \multirow{2}{*}{54} & $2^{\circ}$ & 6,6 & 49,7 & 34,1 & 9,6 & 0 & 2 & 2,5 & 0,8 \\
\hline & $4^{\circ}$ & 5,7 & 26,7 & 46,4 & 19,7 & 1,6 & 3 & 2,9 & 0,9 \\
\hline
\end{tabular}


En síntesis, se aprecia un progreso tímido, dado que la comparación entre medias globales solo aventaja en 0,4 puntos $\left(2,4\right.$ en $2^{\circ}$ curso frente al 2,9 en $4^{\circ}$ ). La moda también experimenta un avance desde el valor 2 (dominio insuficiente), obtenido por el alumnado en $2^{\circ}$ curso, hasta el valor 3 (dominio suficiente) que obtiene en $4^{\circ}$.

\section{Resultados sobre la competencia para desenvolverse como tutores}

Mayoritariamente, las respuestas sobre esta competencia se concentraron en el valor (2), que representa un dominio insuficiente, tanto para la moda como para la media que se aproxima a ese mismo valor, salvo el ítem 58 cuya media es 2,5 , aunque su moda se sitúa en el valor 2 , evidentemente con un porcentaje menor que los anteriores, lo que se justifica por la mayor dispersión de respuestas en este ítem, como refleja su desviación típica, que en cualquier caso no resulta elevada (tabla 9). En efecto, la media global asciende a 2,3 y la moda en todos los casos es 2, luego puede inferirse un dominio insuficiente de esta competencia.

En $2^{\circ}$, los alumnos han valorado su competencia para ejercer la tutoría con una moda insuficiente y unas medias que se reparten entre insuficientes (items 55,57 y 60 ) e insuficientes y suficientes (items 56, 58 y 59), con la única apreciación de que el ítem
59 ha alcanzado un porcentaje para el valor modal (43,2\%) que apenas se distancia del porcentaje de aparición del valor superior a él (41,6\%), como se observa en la tabla 10. En cualquier caso, el valor medio general es 2,3 y el valor modal es en todos los casos insuficiente (2), observándose niveles de dispersión de las respuestas relativamente bajos.

De otra parte, en $4^{\circ}$ se aprecia un matiz diferenciador, puesto que todas las dimensiones que constituyen la competencia como tutor han obtenido una valoración modal suficiente (3) y una media que tiende hacia ese valor $(2,7)$, aunque los valores anteriores al modal (2) también muestran porcentajes elevados de elección, muy similares al modal, en especial el ítem 57, cuyos valores resultan casi idénticos (35,8 y $35 \%$, respectivamente). Puede admitirse, por tanto, un desarrollo tímidamente aceptable de esta competencia.

El leve incremento de la competencia para el ejercicio de la tutoría apenas se respalda por 0,4 puntos de diferencia entre las medias globales de los alumnos de $2^{\circ}$ y $4^{\circ}$, a favor de estos últimos, y en un punto (del dominio insuficiente al suficiente) si se consideran los valores más frecuentes representados por las modas y sus porcentajes. La dispersión es menor entre el alumnado de $2^{\circ}$ curso, aunque en ambos casos es ínfima.

Tabla 9. Resultados globales sobre la habilidad como tutores

\begin{tabular}{|c|c|c|c|c|c|c|c|c|c|}
\hline \multirow{2}{*}{ Ítem } & \multicolumn{4}{|c|}{ Frecuencia de valores } & \multicolumn{2}{c|}{ Tendencia/Dispersión } & \multicolumn{2}{c|}{ Preferencias } \\
\cline { 2 - 11 } & 1 & 2 & 3 & 4 & 5 & Media & Desviación & Moda & $\%$ \\
\hline 55 & 28 & 73 & 39 & 7 & 1 & 2,2 & 0,8 & 2 & 49,3 \\
\hline 56 & 15 & 74 & 43 & 16 & 0 & 2,4 & 0,8 & 2 & 50 \\
\hline 57 & 26 & 65 & 47 & 9 & 1 & 2,3 & 0,8 & 2 & 43,9 \\
\hline 58 & 20 & 59 & 47 & 18 & 4 & 2,5 & 1 & 2 & 39,9 \\
\hline 59 & 18 & 72 & 35 & 23 & 0 & 2,4 & 0,9 & 2 & 48,7 \\
\hline 60 & 29 & 62 & 47 & 9 & 1 & 2,3 & 0,9 & 2 & 41,9 \\
\hline Total & & & & & & 2,3 & & 2 & \\
\hline
\end{tabular}


ISSN 0123-1294 | Educ.Educ. Vol. 18. No. 2 | Mayo-Agosto de 2015 | pp. 209-225.

Universidad de La Sabana | Facultad de Educación

Tabla 10. Resultados por cursos sobre la habilidad como tutores

\begin{tabular}{|c|c|c|c|c|c|c|c|c|c|}
\hline \multirow{2}{*}{ Ítem } & \multirow{2}{*}{ Curso } & \multicolumn{5}{|c|}{ Porcentaje de valores ( 1 a 5 ) } & \multicolumn{3}{|c|}{ Tendencia/Dispersión } \\
\hline & & 1 & 2 & 3 & 4 & 5 & Moda & Media & Desviación \\
\hline \multirow{2}{*}{55} & $2^{\circ}$ & 11 & 45,1 & 39,3 & 4,6 & 0 & 2 & 2,4 & 0,6 \\
\hline & $4^{\circ}$ & 7,3 & 32,6 & 36,4 & 22,6 & 1,1 & 3 & 2,8 & 0,9 \\
\hline \multirow{2}{*}{56} & $2^{\circ}$ & 8,5 & 49,9 & 32,6 & 9,1 & 0 & 2 & 2,3 & 0,8 \\
\hline & $4^{\circ}$ & 7,8 & 34,5 & 38,8 & 18,1 & 0,8 & 3 & 2,7 & 0,8 \\
\hline \multirow{2}{*}{57} & $2^{\circ}$ & 16 & 45,1 & 36,2 & 2,7 & $\mathrm{O}$ & 2 & 2,3 & 0,6 \\
\hline & $4^{\circ}$ & 8,6 & 35 & 35,8 & 19,1 & 1,3 & 2 & 2,7 & 0,9 \\
\hline \multirow{2}{*}{58} & $2^{\circ}$ & 11 & 50,7 & 25,6 & 11,4 & 1,3 & 2 & 2,4 & 0,8 \\
\hline & $4^{\circ}$ & 5,4 & 31,8 & 38,3 & 22,9 & 1,6 & 3 & 2,8 & 0,9 \\
\hline \multirow{2}{*}{59} & $2^{0}$ & 6,9 & 43,2 & 41,6 & 8,3 & 0 & 2 & 2,5 & 0,7 \\
\hline & $4^{\circ}$ & 8,1 & 32,6 & 37,2 & 21 & 1,1 & 3 & 2,7 & 0,9 \\
\hline \multirow{2}{*}{60} & $2^{\circ}$ & 15,2 & 54,5 & 25,2 & 4,2 & 0,8 & 2 & 2,2 & 0,8 \\
\hline & $4^{\circ}$ & 7 & 35,6 & 40,4 & 16,4 & 0,5 & 3 & 2,7 & 0,8 \\
\hline
\end{tabular}

\section{Conclusiones}

Se ha llevado a cabo un estudio descriptivo con estudiantes españoles del grado de Magisterio (mención en EE) para averiguar cuál es su percepción acerca de la formación en competencia comunicativa. Sin duda, la conclusión general del estudio es que los futuros maestros de EE admiten un dominio insuficiente de esta competencia. Así lo corroboran los cálculos realizados que arrojan una media general de 2,5 y un valor modal predominante de 2 . Este dato es consistente con los resultados de otras investigaciones realizadas con estudiantes de la diplomatura de Magisterio (Domingo et al., 2010a, 2010b, 2010c) y de otras licenciaturas (Conchado y Carot, 2013). Igualmente escaso resulta el desarrollo que experimenta esta competencia durante el transcurso de la carrera, como ya demostraron otros estudios (Domingo et al., 2010a; 2010b; 2010c). Los alumnos próximos a egresar obtienen un dominio ligeramente superior en sus habilidades comunicativas y se muestran preocupados a la hora de desarrollar una comunicación eficaz en sus futuros centros educativos. Este testimonio es alarmante, si se tiene en cuenta que los informes legislativos y los modelos teóricos expertos otorgan a esta com- petencia un valor crucial para el desempeño profesional docente (Camacho y Sáenz, 2000; Perrenoud, 2004; Pérez, 2008).

En general, se aprecia una autovaloración deficitaria del alumnado en la mayoría de las subdimensiones que incluye la competencia comunicativa. En efecto, los sujetos de la muestra consideran insuficientes sus habilidades como emisores docentes y sus destrezas para manejarse eficazmente en las reuniones, así como para comunicar de forma pertinente en la sala de clase, lo cual coincide con los resultados obtenidos en otros estudios realizados con estudiantes de diplomatura (Domingo et al., 2010a, 2010b, 2010c), licenciatura (Conchado y Carot, 2013) y grados (Peñalva, López y Landa, 2013).

Tampoco poseen una buena percepción acerca de su competencia para el desarrollo de las funciones tutoriales, algo que ya constató otro estudio cuando puso de manifiesto que uno de los bloques de competencias en las que el profesorado requiere de más formación es el que hace referencia a la tutoría (Álvarez, Romero, Gil, Rodríguez, Clares et al., 2011). 
Todos estos datos son consistentes con la opinión que tienen los propios profesores universitarios de Magisterio, quienes desconfían del nivel de logro alcanzado por sus alumnos en el dominio de la competencia comunicativa (Domingo et al., 2010c; Vine y Ferreira, 2102; Domingo, Gallego y Rodríguez, 2013) y lamentan su falta de inclusión reglada en los nuevos planes de estudios.

No obstante, los sujetos encuestados entienden que sus habilidades como receptores son suficientes, lo que concuerda con los datos obtenidos por Domingo et al. (2013) en un estudio realizado con profesores universitarios. Este hecho resulta muy curioso por ser coherente con el modelo docente universitario tradicional (lección magistral), en el cual es notorio el papel pasivo del alumno como receptor de la información del profesor.

Sin embargo, en este nuevo contexto, los futuros egresados necesitan una formación inicial centrada en el desarrollo de competencias profesiona- les docentes (especialmente las comunicativas), que solo podrá alcanzarse si se realizan estudios diagnósticos que permitan detectar deficiencias para implementar los cambios oportunos. De ahí la necesidad de promover metodologías que favorezcan el aprendizaje comunicativo de los estudiantes, generar climas de diálogo que posibiliten la expresión libre, propiciar aprendizajes comunicativos aplicables a situaciones complejas, problematizar los discursos tratando de descubrir presuposiciones y prejuicios, estimular constantemente la capacidad de reflexionar de los futuros docentes, etc.

Pero como toda investigación, este estudio no está exento de limitaciones, como por ejemplo su carácter descriptivo, el número de participantes implicados o su localismo. Futuras investigaciones deberían dirigirse a validar nuevos instrumentos que permitan un análisis más pormenorizado de la competencia comunicativa de los futuros docentes, o a valorar la eficacia de programas de formación específicos.

\section{Referencias}

Álvarez, V., Romero, S., Gil, J., Rodríguez, J., Clares, J. et al. (2011). Necesidades de formación del profesorado para la adaptación de su docencia al Espacio Europeo de Educación Superior (EEES). Relieve, 17 (1), 1-22. Recuperado el 19 de enero de 2014 de http://www.uv.es/RELIEVE/v17n1/RELIEVEv17n1_1.htm.

Camacho, S. y Sáenz, Ó. (2000). Técnicas de comunicación eficaz para profesores y formadores. Alcoy: Marfil.

Camargo-Escobar, I. M. y Pardo-Adames, C. (2008). Competencias docentes de profesores de pregrado: diseño y validación de un instrumento de evaluación. Universitas Psychologica, 7 (2), 441-455.

Clemente, J. S. y Escribá, C. (2013). Análisis de la percepción de las competencias genéricas adquiridas en la universidad. Revista de Educación, 362, 535-561.

Conchado, A. y Carot, J. M. (2013). Puntos fuertes y débiles en la formación por competencias según los graduados universitarios españoles. Revista de Docencia Universitaria, 11 (1), 429-446.

Díaz Barriga, F. (2006). Enseñanza situada: vínculos entre la escuela y la vida. México: McGraw-Hill Interamericana. 
ISSN 0123-1294 | Educ.Educ. Vol. 18. No. 2 | Mayo-Agosto de 2015 | pp. 209-225.

Universidad de La Sabana | Facultad de Educación

Díaz Barriga, F. (2010). Los profesores ante las innovaciones curriculares. Revista Iberoamericana de Educación Superior, 1 (1), 37-57.

Domingo, J., Gallego, J. L., García, I. y Rodríguez, A. (2010a). Competencias comunicativas del maestro en formación. Profesorado. Revista sobre currículum y formación del profesorado, 14 (2). Recuperado el 19 de enero de 2014 de http://www.ugr.es/ recfpro/rev142COL7.pdf

Domingo, J., Gallego, J. L., García, I. y Rodríguez, A. (2010b). La competencia comunicativa en alumnos de la especialidad de Lengua Extranjera de Magisterio de la Universidad de Granada. Enseñanza \& Teaching, 28, 139-160.

Domingo, J., Gallego, J. L., García, I. y Rodríguez, A. (2010c). Análisis de las percepciones del profesorado y del alumnado de educación especial sobre sus habilidades comunicativas. Revista de Educación Inclusiva, 3 (2), 47-64.

Domingo, J., Gallego, J. L. y Rodríguez, A. (2013). Percepción del profesorado sobre competencia comunicativa en estudiantes de magisterio. Perfiles Educativos, 35 (142), 54-74.

Ferreiro, R. F. (2011). Tres vértices del triángulo de las competencias didácticas: teoría, metodología y método. Revista Complutense de Educación, 22 (1), 11-23. Recuperado el 25 de marzo de 2014 de http://dx.doi. org/10.5209/rev_RCED.2011.v22.n1.1

Fox, D. J. (1981). El proceso de investigación en educación. Pamplona: Eunsa.

Gutiérrez, D. (2011). Desarrollo de competencias mediante métodos didácticos. En Jaik, A. y Barraza, A. (coords.). Competencias y educación. Miradas múltiples de una relación (pp. 163-179). México: Instituto Universitario Anglo Español A.C.y Red Durango de Investigadores Educativos A.C.

Latorre, A., Del Rincón, D. y Arnal, J. (1996). Bases metodológicas de la investigación educativa. Barcelona: Gràfiques, S.A.

Marciales-Vivas, G. P., González-Niño, L., Castañeda-Peña, H. y Barbosa-Chacón, J. W. (2008). Competencias informacionales en estudiantes universitarios: una reconceptualización. Universitas Psychologica, 7 (3), $643-654$.

Martínez, C. (2012). Estadística y muestreo. Bogotá: Ecoe Ediciones.

McMillan, J. H. y Schumacher, S. (2005): Investigación educativa: una introducción conceptual. Madrid: Pearson Addison Wesley.

Mérida, R. (2013). La controvertida aplicación de las competencias en la formación docente universitaria. Revista de Docencia Universitaria, 11 (1), 185-212.

Peñalva, A., López, J. y Landa, N. (2013). Competencias emocionales del alumnado de Magisterio: posibles implicaciones profesionales. Revista de Educación, 362, 690-712. 
Pérez García, M. P. (2008). Competencias adquiridas por los futuros docentes desde la formación inicial. Revista de Educación, 347, 343-367.

Perrenoud, P. (2004). Diez nuevas competencias para enseñar. Barcelona: Graó.

Tobón, S. (2012). El enfoque socioformativo y las competencias: ejes clave para transformar la educación. En Tobón, S. y Jaik, A. (coords.). Experiencias de aplicación de las competencias en la educación y el mundo organizacional (pp. 3-31). México: Red Durango de Investigadores Educativos A.C.

Vine, A. y Ferreira, A. (2012). Mejoramiento de la competencia comunicativa en español como lengua extranjera a través de la videocomunicación. Revista de Lingüistica Teórica y Aplicada, 50 (1), 139-160. 